\title{
EVALUACIÓN DE LA VIABILIDAD DEL USO DE LOS MÉTODOS DE VALORACIÓN ECONÓMICO-AMBIENTAL EN UN CONTEXTO ESPACIAL
}

\section{Feasibility Evaluation of Spatial use of economic-environmental valuation methods}

\author{
Inmaculada Gómez Jiménez, Pablo Martínez de Anguita, \\ Raúl Romero Calcerrada
}

Recibido 17/10/2006; aceptado el 20/01/2007

Resumen. Los métodos de valoración económico-ambiental son herramientas útiles de cara a la integración objetiva de los tres aspectos del territorio: ecológico, económico y social. Basándose en las reglas de mercado valoran en unidades monetarias los tres aspectos, para así hacerlos comparables tanto en el espacio como en el tiempo.

Sin embargo sus resultados, hasta el momento apenas han podido ser cartografiados $y$, por tanto, no han sido utilizados en la planificación en un contexto espacial.

El presente artículo trata de evaluar cuáles son las principales causas de que ocurra esta situación de incompatibilidad entre los métodos y su aplicación espacial, así como las posibilidades de adaptación que permitirían solventarla.

Las estimaciones de valor de no-uso, o de los aspectos no tangibles como la conservación para las generaciones futuras de una determinada especie no pueden ser cartografiadas salvo quizá a una escala global. Otros aspectos ambientales podrían ser cartografiados con adaptaciones concretas que aún están por desarrollar.

Palabras clave: economía ambiental, uso espacial, escala, Valor Económico Total

Abstract. The economic-environmental valuation methods are useful tools to achieve the integration of the three aspects on spatial planning: ecological, economic and social. They value the three aspects in monetary units, following the market rules, to make them comparable as much spatially as temporally.

However, their results cannot be mapped at now, and therefore they cannot be used in the planning in a spatial context.

This paper evaluates the main causes of the incompatibility between the methods and its spatial application, as well as the possibilities of adaptation that would allow over it.

Currently, estimations of no-use value, non-tangible aspects, as the preservation for the future generations of certain species, cannot be mapped except maybe in a globe scale. However, other environmental aspects could be mapped with certain adaptations that are still to be developed.

Key Words: environmental economics, spatial use, scale, Total Economic Value 


\section{INTRODUCCIÓN}

La planificación del territorio es la base para una gestión y desarrollo adecuados. Tras la llamada Cumbre de Río en 1992, el concepto de desarrollo sostenible pasó a recibir una mayor atención en la planificación territorial. El objetivo del planificador pasa a partir de entonces de ser alcanzar el desarrollo económico máximo a lograr un desarrollo sostenible. Para lograr este desarrollo sostenible se hace indispensable considerar el aspecto ambiental en la gestión y en la toma de decisiones, y por tanto también en la planificación del territorio.

Esta incorporación del medio ambiente en la planificación se ha visto facilitada y potenciada por la disponibilidad de información temática suministrada por la teledetección y el apoyo de los Sistemas de Información Geográfica (SIG). Así el desarrollo de la planificación física ha sido muy importante en los últimos años.

Este gran desarrollo ha motivado nuevas inquietudes hacia la mejora de la planificación territorial. El medio ambiente no se contempla ya como un elemento a conservar, sino como un factor que interacciona con el resto de elementos que se desarrollan sobre el territorio. Por esto es creciente la búsqueda de integración en la planificación del medio natural con el medio socioeconómico, tratándose de considerar las interacciones entre los tres aspectos del territorio. Para alcanzar esta integración el método más utilizado en la planificación territorial han sido las evaluaciones multicriterio. Sin embargo esta metodología establece de forma más o menos subjetiva la importancia o la relación entre los distintos elementos o factores. La subjetividad de estas decisiones se reduce significativamente si los tres aspectos son medidos en unidades comparables.

Hasta el momento, el desarrollo más ambicioso hacia la integración de los distintos aspectos lo ha realizado la economía ambiental, proponiendo la transformación de los valores ambientales a unidades monetarias. Esta transformación parte de una hipótesis básica, calcular el valor real económico, y no el que desde otra perspectiva moral, ecológica o social debería tener un bien. Así, desde la teoría económica clásica, no se valora el bien o servicio ambiental per se sino el valor que tiene para el consumidor el servicio ambiental recibido, expresado en términos monetarios. El valor del bien o servicio ambiental, percibido por el consumidor es, por tanto, la cantidad que éste está dispuesto a pagar por acceder, mantener o mejorar un aspecto del ambiente. De este modo la cuantificación económica de un valor ambiental queda acotada por la disponibilidad a pagar de los consumidores. Esta cuantificación puede tener o no una relación directa con la importancia de dicho valor ambiental como función o ecosistema en el presente o en el futuro.

A pesar del creciente desarrollo de los métodos de valoración, su aplicación se ha realizado casi exclusivamente en áreas concretas (p.e. para calcular el valor de conjunto de bosques (Tyrvainen, 2001)) y para analizar determinadas actuaciones hipotéticas (p.e. disposición a pagar por un programa de prevención de incendios (Riera y Mogas, 2004)). Esta concreción, que ofrece grandes ventajas de cara a la calidad de los resultados, tiene un valor meramente informativo en la planificación territorial. Esto se debe a que los métodos, sobre todo la valoración contingente, suponen elevados costes que no se justifican para su uso en un único proceso de decisión pero que serian inabordables en una planificación total de un territorio. La utilización de estas técnicas de valoración implicaría generar, de cara a la planificación, una valoración para cada zona y para cada actividad posible a desarrollar, lo cual es temporal y económicamente inabordable e ineficiente. La cantidad de aspectos que han de ser considerados en una planificación territorial, con diferentes matices sectoriales y de escala, no puede ser abordada en la práctica mediante estos métodos de modo económicamente eficiente.

Así, en el uso de la valoración económica de bienes y servicios ambientales en la planificación territorial existen dos elementos clave: la integración (agregación) entre valores y su disposición espacial. La agregación entre valores es clave cuanto se utiliza un sistema como el SIG de disposición en capas. A menudo es muy complejo delimitar los bienes 0 servicios a valorar de modo que no induzcan a una doble estimación. Por ejemplo, cuando se utiliza la valoración contingente, es muy complejo determinar qué parte de la valoración corresponde a cada bien o servicio, e incluso desagregar los valores de opción o no-uso de los de uso. Por tanto la superposición directa de elementos para su análisis puede conllevar dificultades.

Sin embargo, si se dispusiese de una cartografía de valores económico-ambientales suficientemente individualizados, esta información podría utilizarse en la planificación como se hace actualmente con la disposición de las infraestructuras, uso del suelo, etc. Y así estimarse los bienes o servicios afectados y obtener el análisis coste-beneficio de las inversiones en la zona. Es más, podría distinguirse entre beneficios privados (realmente ya manifestados monetariamente en la economía y que por tanto perciben los propietarios o usufructuarios) y beneficios sociales (también Ilamados externalidades).

Sin embargo no existen cartografías de este tipo. Aunque se han realizado intentos parciales, actualmente los métodos de valoración económica no se han adaptado al formato cartográfico (Martínez de Anguita, 2004). En muchos casos el diseño del método de valoración o sus aplicaciones son directamente incompatibles con esta adaptación y en otros es sumamente complejo.

Para incorporar la economía como elemento cartográfico en la planificación es necesario en primer lugar analizar los problemas (de cara a su uso espacial) inherentes a la na- 
turaleza del bien o servicio y posteriormente considerar las peculiaridades de los métodos existentes de valoración económico-ambiental.

\section{ECONOMÍA AMBIENTAL}

La economía ambiental identifica los fallos del mercado (externalidades, bienes comunes...) como causantes de los problemas ambientales y plantea como solución a estos problemas la internalización de los bienes y servicios ambientales en el sistema de mercado. Así, ha desarrollado diversas técnicas que permiten la valoración monetaria de los bienes y servicios ambientales, que originariamente carecen de precio de mercado, para que éstos puedan compararse con aquellos bienes y servicios que sí disponen de un precio de mercado.

En el mercado los consumidores establecen sus niveles de preferencias mediante el precio. En los métodos de valoración económica de bienes y servicios ambientales podemos establecer a partir de las preferencias de la población un valor económico, comparable al resto. Asumen no sólo que el consumidor está dispuesto a asignar un valor económico a estos bienes y servicios ambientales, sino también que tiene capacidad suficiente para hacerlo. Estas dos premisas son de dudoso cumplimiento cuando la valoración se centra en cuestiones que escapan a la concepción utilitarista del individuo, es decir los valores de no-uso. Por otro lado es importante situar la valoración económica en un contexto adecuado: no tiene mucho sentido comparar el valor de dos espacios naturales hallados uno en Europa y otro en un área remota de un pais en desarrollo por sus estimaciones económicas de uso o recreo, donde las preferencias de los habitantes y su nivel de renta apuntan previamente hacia la supervivencia. Esta aclaración espacial, como es lógico, tiene su equivalente temporal. Esta misma incongruencia se da- ría si comparásemos una valoración del mismo sitio en la Edad Media con el momento actual.

Sin embargo, es indudable que aún cuando la valoración sea parcial, la economía ambiental ofrece herramientas muy valiosas para la planificación y la toma de decisiones. La posibilidad de disponer de medidas cuantitativas y conmensurables en todos los ámbitos a considerar en la planificación, simplifica y acelera la toma de decisiones.

\section{BIENES Y SERVICIOS AMBIENTALES}

Aún partiendo de la premisa de que incluso la valoración parcial es útil a la ordenación territorial, la clave de la integración entre los aspectos ecológico, económico y social sólo se alcanza con la valoración económica total (VET) (ver tabla 1). La valoración económico-ambiental en el espacio implica la localización (georreferenciación), directa o indirecta, de bienes servicios y de sus precios en un momento temporal determinado.

\subsection{Valores de uso directo}

Comenzando de menor a mayor complejidad, los valores de uso directo son en su mayoría tangibles: madera, pasto... y es habitual que tengan un precio de mercado. La espacialización de estos valores es directa, sólo se requiere una cartografía de base que permita calcular crecimientos para distinguir entre capital y renta de cara a la valoración. El precio se obtendrá por diferentes vías, más o menos directas. Por ejemplo el valor en pastos si no está tasado como tal puede inferirse de un valor equivalente en forraje.

Sin embargo, la obtención y utilización de los precios en la elaboración de las cartografías tampoco es inmediata. La dificultad en el caso de los precios estriba en que cuando la escala se hace más detallada se hace más complejo encon-

TABLA 1. Tipos de valores considerados por la teoría del valor económico total (Pearce y Moran, 1994; Pearce y Turner, 1991)

\begin{tabular}{|c|c|c|c|}
\hline \multicolumn{2}{|c|}{ Tipologías de valores } & Definición & Ejemplos \\
\hline \multirow{2}{*}{ Valores de Uso } & Directo & $\begin{array}{l}\text { Se realiza un uso normalmente consuntivo y } \\
\text { con rivalidad. }\end{array}$ & $\begin{array}{l}\text { Madera, agricultura, pastos, recreo, caza } \\
\text { у pesca, ... }\end{array}$ \\
\hline & Indirecto & $\begin{array}{l}\text { Forman parte de la función de producción de } \\
\text { otros bienes o servicios }\end{array}$ & $\begin{array}{l}\text { Sumidero de } \mathrm{CO}_{2} \text {, mantenimiento del sue- } \\
10, \ldots\end{array}$ \\
\hline \multicolumn{2}{|l|}{ Opción } & $\begin{array}{l}\text { No existe uso actual pero se valora que el uso } \\
\text { pueda realizarse en un futuro }\end{array}$ & $\begin{array}{l}\text { Futuras fuentes de alimentos, medicinas, } \\
\text {... }\end{array}$ \\
\hline \multirow{2}{*}{ Valores de no-uso } & Legado & $\begin{array}{l}\text { Conservación del bien o servicio para futuras } \\
\text { generaciones }\end{array}$ & $\begin{array}{l}\text { Conservación de paisajes singulares, pre- } \\
\text { vención cambio climático, ... }\end{array}$ \\
\hline & Existencia & Mantenimiento perse & $\begin{array}{l}\text { Conservación de especies en peligro de } \\
\text { extinción, hábitats amenazados, ... }\end{array}$ \\
\hline
\end{tabular}

Elaboración propia Fuente: (Azqueta Oyarzun, 2002; Merlo y Croitoru, 2005) 
trar un precio ajustado. Normalmente se dispone de precios a nivel nacional, promediados para un año, existiendo variaciones considerables, según los casos dependiendo de las escalas temporales y espaciales. De hecho, los precios de estos bienes, marcadamente territoriales, tienen una fuerte dependencia de la localización. Por ejemplo los precios agrícolas tienen una fuerte dependencia según la existencia y disponibilidad cercana de infraestructuras de venta, de mercado, de transporte y de las economías de escala. Todas estas cuestiones pueden llegar a ser muy significativas. Valga como ejemplo el detallado estudio de accesibilidad que se requiere en los estudios de implantación de centrales de aprovechamiento energético de biomasa, donde el precio del combustible es directamente proporcional a la distancia de trasporte.

Merecen mención en este apartado dos aspectos muy importantes y habituales: la caza y el recreo. Son valores de uso directo pero no podrían clasificarse en principio como tangibles.

La caza, y de modo equivalente la pesca deportiva, es una actividad lúdica y dinámica. Su valoración debe centrarse en los territorios aptos para la caza y en la cantidad de piezas que es posible capturar por cada cazador en esa zona. Hasta donde es una práctica regulada, legislada, la cartografía puede generarse a partir de los datos de los cotos de caza (o en su caso pesca), disponiendo de datos anuales de productividad del coto y de cazadores que hacen uso de éstos. Las licencias de caza, por ejemplo, que son un valor más del precio de la caza no pueden ser correctamente incluidas en la cartografía con los datos actuales a una escala infraregional o incluso nacional ya que la licencia se liga a la Comunidad Autónoma y no existen datos sobre el uso concreto que realiza cada cazador en cada una de las diferentes zonas de caza.

El recreo es uno de los servicios ambientales más estudiados por la economía ambiental (Millward, 1993; Parsons y Keraly, 1994; Pukkala et al., 1995; Tyrvainen, 2001; Tyrvainen y Vaananen, 1998). Si bien es relativamente sencillo georreferenciar los puntos de uso recreativo, las valoraciones se centran habitualmente sobre un conjunto: un bosque, un área significativa o protegida... La cuestión es ¿cuánta superficie es realmente necesaria para que el valor del uso en el punto (o línea en caso de senderos) no varíe? ¿Cuál es la relación entre el uso recreativo y el paisaje? Es decir, ¿cuál es el área de influencia de un área recreativa? A pesar de que existen trabajos que intentan relacionar elementos del territorio a diferentes valoraciones (Beneitez López et al., 2006; Oglethorpe et al., 2000) éstas son cuestiones aún no resueltas y cuya resolución es indispensable para que la elaboración de cartografías sea correcta.

El caso del paisaje presenta una gran peculiaridad desde el punto de vista espacial, fundamentalmente cuando lo que se plantean son escenarios de cambio. El paisaje no tie- ne valor únicamente por las coberturas que lo componen 0 las infraestructuras que se le interponen. Aún cuando cuenca visual y espectador no cambien, dos paisajes con la misma cobertura en superficie, e incluso porcentaje dentro de la visual, pueden no tener el mismo valor. Esto es debido a que el valor del paisaje tiene una alta dependencia de cuestiones como elementos de borde, efectos mosaico, linealidad, textura, heterogeneidad, singularidad... Todos estos aspectos no son fácilmente considerables en un análisis aislado de paisaje, pero en un SIG pueden cobrar un amplio significado y facilitar la replicabilidad de las valoraciones.

\subsection{Valores de uso indirecto}

Muchos de los aspectos ambientales (podría afirmarse que todos sin un gran margen de error) tienen repercusiones en la función de producción de otros bienes o servicios. La calidad del aire afecta al crecimiento de los cultivos, la erosión a la productividad del suelo y a la productividad piscicola, la biodiversidad es un aspecto clave en el control de plagas y enfermedades...

La medida en que estos valores de uso indirecto pueden ser cartografiados depende de dos aspectos clave: el grado de "inmovilidad territorial" y los datos disponibles. Por ejemplo para evaluar los efectos de la calidad del aire en los cultivos necesitariamos conocer datos de emisión de contaminantes y las redes de distribución espacial, que son cambiantes en una escala temporal muy pequeña. De este modo el tipo de cartografía dependerá de la disposición de una red de medida constante de contaminantes y flujos de viento, mostrándose en una cartografía digital de actualización continua.

La relación con la función de producción de estos valores implica siempre una mayor complejidad, con respecto a los valores de uso directo, en la obtención de los datos necesarios para la elaboración de la cartografía. Para establecer la función de producción o la relación causa efecto es necesario un modelo que permita observar variaciones en los bienes o servicios cuando varía la variable a considerar. Por ejemplo, necesitamos un modelo de erosión para poder estimar las pérdidas de productividad en cultivos se producen en función de las diferentes coberturas o condiciones climáticas. Necesitamos cuantificar la variable y su relación sobre los bienes y servicios de uso que se ven afectados. En el caso de la erosión, no sólo necesitamos saber con exactitud dónde y en qué medida se produce (Gómez Jiménez et al., 2006), sino dónde van a parar esos sedimentos y cómo afecta a la calidad del agua o de suelo donde se depositan.

Puesto que hablamos de funciones de producción, un aspecto muy importante a considerar son los puntos críticos. Puntos de inflexión a partir de los cuales las condiciones se vuelven indeseables o irreversibles (Ekins, 2003; Farber et al., 2002). La valoración tras superar el punto crítico es significativamente diferente, tanto en cantidad como en método 
(Azqueta, 2002). Sin embargo, pese a su importancia teóri$c a$, en la práctica no existen trabajos orientados a la evaluación de estos puntos críticos, salvo algunas aproximaciones teóricas (Ekins, 2003; van Noordwijk et al., 2004).

\subsection{Valores de opción}

Aunque en la teoría económica estos valores de opción no se consideren como valores de uso, en la práctica de la elaboración de cartografías no requieren distinción alguna. Únicamente se requiere información sobre cuáles de los recursos de uso (directo o indirecto) están siendo realmente utilizados y cuáles podrian serlo en el futuro. Si bien no tienen ninguna particularidad desde el punto de vista espacial. Representan el stock de valores no utilizados pero utilizables en un futuro.

\subsection{Valores de no-uso: valores de legado y valores de existencia}

Los valores de no uso pueden clasificarse en valores de legado y valores de existencia (ver tabla 1). Dado que la diferencia entre ambos conceptos no plantea dificultades metodológicas de cara a su cartografía, se muestran agrupados en este apartado. Las dificultades que presenta su valoración no son superiores a las que presenta su cartografía. Son valores abstractos en su mayoría y muy dependientes de cuestiones como la escasez de recursos presente $y / 0$ futura (Batabyal et al., 2003; Drechsler, 2004; Jayasuriya, 2003).

Algunos estudios ponen de manifiesto la importancia que presenta el ámbito geográfico que se le da al estudio (Prada Blanco et al., 2005; Riera y Mogas, 2004). Las implicaciones espaciales de esta relatividad son evidentes. No es posible valorar un elemento aislado para representarlo en una cartografía. El cálculo debe hacerse entonces sobre cuestiones teóricas: mantenimiento de la biodiversidad, conservación de un espacio protegido, evaluación de daños morales por un vertido...

No sólo importa la dimensión espacial y localización de lo que se valora, sino la dimensión espacial y localización de quiénes valoran. Es decir, la selección de la población implicada en la valoración.

Supongamos que lo que se plantea valorar es la supervivencia del águila imperial ibérica como especie en peligro de extinción. Presenta un valor de legado (queremos que las generaciones futuras puedan disfrutar de su existencia) y un valor de existencia como ente en sí mismo. Si se elabora una cartografía de su población actual el valor de preservación ¿estará ligado a toda la población? Podría decirse que sólo a la población necesaria para la supervivencia de la especie, pero entonces ¿qué parte de la población total sería representada como tal? El hecho es que cuando valoramos la pervivencia de la especie no estamos valorando a sus individuos, ni sus hábitat ni sus poblaciones, sino que se realice lo necesario para su mantenimiento en conjunto. Y esto se hace más patente cuando se usa una escala detallada (ver figura 1).

Cuando se considera a toda la población (a toda la humanidad) en la valoración sí podemos obtener un valor aproximado para una cartografía global en el que la población sea representada como una superficie en conjunto e indivisible.

Cuando la escala pasa a ser mayor, es necesaria una división de la población valorada (o, en el mejor de los casos, limitamos sólo la consideración de la población que valora). La división de la población valorada no es posible en cuanto no sabemos su importancia ecológica relativa en términos

Figura 1. Representación de los problemas de escala ante los valores de legado. La conservación de una especie no es divisible entre la presencia de ejemplares. Los datos de poblaciones son sólo ilustrativos.

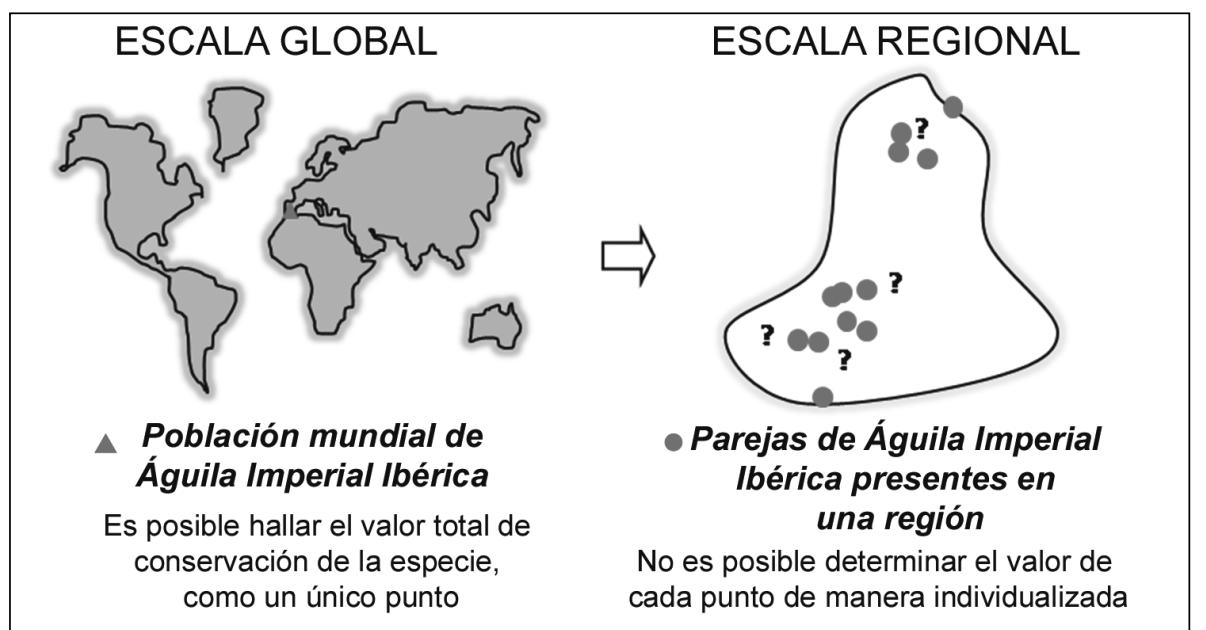

Elaboración propia. 
absolutos. Con lo cual lo podemos expresar bien la cuestión a la población que valora. La división de la población que valora (las personas encuestadas) implica que la valoración obtenida será siempre parcial.

De este modo, podemos estar elaborando la cartografía de un área protegida con presencia de unos cuantos nidos de esta rapaz (ver escala local en figura 1). No podríamos valorarlos en función de lo que la gente valora la pervivencia de la especie sino en cuanto valora la presencia de estos nidos, conociendo la importancia que su existencia tiene en la pervivencia de la especie. Y aqui se plantea una disyuntiva con los métodos de valoración. Los métodos de valoración económica se basan por defecto en que el individuo que ejerce su "voto" de mercado (Azqueta, 2002; Azqueta y Delacamara, 2006) dispone de información suficiente. Y es evidente que este respecto no se cumple, en casi la totalidad de la población, para la gran mayoría de aspectos ambientales de no-uso. Si para los especialistas es muy complejo determinar la aportación que un unidon puede suponer a la conservación de una especie cuánto más complejo le resultará al ciudadano de a pie.

Pese a todo, existen algunos trabajos al respecto que tratan de "distribuir» valores de conjunto según índices o elementos (Elorrieta y Castellano, 2000; Oglethorpe et al., 2000). Hasta el momento las valoraciones de bienes y servicios de no-uso son únicamente factibles utilizando el méto- do de valoración contingente. Este método se ha aplicado a toda una zona o se ha transferido (benefit transfer) de otra parte, pero siempre realizado para toda la zona. Esto entra en conflicto con cualquier cartografía a escala local o regional, que debe diferenciar valores entre diversas localizaciones y no debe ser uniforme.

\section{MÉTODOS DE VALORACIÓN ECONÓMICO-AMBIENTAL}

Existe una gran diversidad de métodos de valoración económico-ambiental. Cada metodología está diseñada para una serie de aplicaciones y se basa en diferentes supuestos de la economía ambiental. Existen métodos que obtienen sus valores de modo indirecto a través del mercado ya existente, como el método del coste de viaje. Otros métodos como los basados en la función de producción o los costes de reposición utilizan los valores del mercado de un modo directo. Mientras, la valoración contingente o los experimentos de elección (variante de la valoración contingente) generan mercados hipotéticos.

En la tabla 2 se muestran los principales métodos, con sus correspondientes ámbitos de aplicación (tipos de valores que pueden considerar) y una breve descripción de las fundamentales limitaciones teóricas que presentan. Las limitaciones en el ámbito de aplicación (no todos los métodos pue-

TABLA 2. Principales métodos de valoración económica de bienes y servicios ambientales (Azqueta, 2002; Croitoru, 2004; Pagiola et al., 2004). Se indica su ámbito de aplicación y sus principales limitaciones teóricas.

\begin{tabular}{|c|c|c|}
\hline Metodología & Aplicaciones & Limitaciones teóricas \\
\hline $\begin{array}{l}\text { Función de producción (cam- } \\
\text { bios en la productividad) }\end{array}$ & $\begin{array}{l}\text { Valoración impactos en bienes ambientales que for- } \\
\text { man parte directa de alguna función de producción. }\end{array}$ & $\begin{array}{l}\text { Generalmente se carece de datos sobre el cam- } \\
\text { bio producido en el servicio. }\end{array}$ \\
\hline Impactos sobre la salud & $\begin{array}{l}\text { Cualquier impacto que afecte a la salud (morbilidad } \\
\text { y mortalidad). }\end{array}$ & $\begin{array}{l}\text { Se carece de funciones dosis-respuesta; se su- } \\
\text { bestiman o descartan las preferencias por la sa- } \\
\text { lud y el valor de la vida es de difícil estimación. }\end{array}$ \\
\hline Costes de reposición & $\begin{array}{l}\text { Mide el coste de sustituir o remplazar el bien o servi- } \\
\text { cio por otro. }\end{array}$ & $\begin{array}{l}\text { Tiende a sobreestimar el valor actual. Implica } \\
\text { que el bien o servicio tenga un substituto con } \\
\text { valor de mercado. }\end{array}$ \\
\hline Coste de viaje & Valor recreativo. & $\begin{array}{l}\text { Limitado al valor recreativo } \\
\text { Dificultad en caso de múltiples destinos. }\end{array}$ \\
\hline Precios hedónicos & $\begin{array}{l}\text { Calidad del aire, belleza escénica, beneficios cultura- } \\
\text { les. Cartografias localizadas. }\end{array}$ & $\begin{array}{l}\text { Requiere cantidades excesivas de datos y no } \\
\text { siempre de fácil acceso (transacciones de pro- } \\
\text { piedades). }\end{array}$ \\
\hline $\begin{array}{l}\text { Valoración contingente y ex- } \\
\text { perimentos de elección }\end{array}$ & $\begin{array}{l}\text { Cualquier servicio, incluido el valor de no uso y de } \\
\text { opción. }\end{array}$ & Alto sesgo, baja confiabilidad (Riera, 1992). \\
\hline Transferencia de resultados & $\begin{array}{l}\text { Cualquiera. } \\
\text { Cartografías de valores }\end{array}$ & $\begin{array}{l}\text { Puede ser inexacto. Requiere que los datos de } \\
\text { origen estén muy bien definidos y sean asimila- } \\
\text { bles a los de destino. }\end{array}$ \\
\hline
\end{tabular}

Elaboración propia. 
den contemplar o estimar todos los tipos de bienes o servicios) y las limitaciones teóricas son claves para comprender los problemas que presentan de cara a su utilización para la elaboración de cartografías.

De todos los métodos de valoración económica de bienes y servicios ambientales (tabla 2), el que más fácilmente se adapta a la elaboración de cartografías es el método de transferencia de resultados (benefit transfer). Esto se debe a que para poder realizar la transferencia de los resultados obtenidos con un método de un lugar a otro, es necesaria una función de transferencia (Desvousges et al., 1998) que relaciona determinadas variables de origen y del destino con los valores obtenidos. Conocida esta relación, generar la cartografía depende exclusivamente de disponer de adecuadas cartografías de las variables de origen, no es un problema espacial.

Sin embargo el método de transferencia de resultados es el más difícil de aplicar ya que requiere un meta-análisis (Villa et al., 2002) de un gran conjunto de estudios sobre varios sitios y que mantengan una homogeneidad en su aplicación. La realidad es que el número de estudios es aún reducido en la mayoria de las localizaciones (Chattopadhyay, 2003; Eade y Moran, 1996; Lovett et al., 1997; Oglethorpe et al., 2000).

Además se añade la complejidad de que las valoraciones disponibles se han realizado habitualmente para un área extensa en conjunto sin considerar los elementos separados que permitan fácilmente seleccionar las variables determinantes en la valoración.

Por su parte, el método de precios hedónicos es también un método aplicable al ámbito espacial. Tiene una importante base en las relaciones topológicas. Su aplicación más habitual, el precio de la vivienda respecto a variables ambientales como la calidad del aire o la disponibilidad de áreas verdes permite la elaboración de cartografías urbanas fundamentalmente. Sólo es aplicable a escalas muy detalladas y tiene una elevada dependencia de la disponibilidad de datos.

El resto de métodos presentan problemas ligados al tipo de valores que consideran. Los problemas de coste de viaje ya se comentaron en el apartado 3.1. junto al aspecto recreativo. Al igual que los métodos de función de producción (y de modo equivalente los cambios en la salud, que es una variante) se trataron en el punto 3.2 .

Cartografiar los valores obtenidos mediante el método de los costes de reposición es sencillo. Basta cartografiar el bien a reponer. Sin embargo, es el método con menor fortaleza teórica y con una fuerte tendencia a sobreestimar los valores. La disposición a pagar de un individuo siempre es inferior a la disposición a aceptar aún cuando la cuestión a valorar es la misma (Azqueta, 2002).

Finalmente, el método de valoración contingente y su variante el método de los experimentos de elección son los únicos capaces de considerar los valores de no-uso. A los problemas intrínsecos de la valoración de estos bienes y servicios de no-uso, se suman los propios de los métodos (Bateman et al., 2001; Carson et al., 2001; Clark et al., 2000; Spash, 2000; Venkatachalam, 2004). Plantear un estudio de valoración contingente y aún más de experimento de elección en el que los elementos representables en el territorio fuesen evaluados separadamente es inabordable aún cuando se sostuviera teóricamente.

\section{DISCUSIÓN}

La valoración económica de bienes y servicios ambientales plantea de por sí diversas controversias éticas y/o teóricas. Por no ser el objetivo de este artículo no se ha entrado a considerar las consideraciones éticas que rodean a los métodos. Tampoco se han descrito consideraciones teóricas de base de la economía ambiental, sólo aquellas que afectan a los métodos de valoración y de un modo concreto al objetivo de este artículo. Sin embargo, aporta indudables ventajas de cara a la consideración de los aspectos ambientales en la planificación territorial.

Hasta el momento sólo ha estado disponible para la evaluación de actuaciones concretas, cómo un cambio en el programa de prevención contra incendios (Riera y Mogas, 2004) u otros aspectos puntuales (Elorrieta y Castellano, 2000; Mogas y Riera, 2004). A pesar de estos trabajos, la no disponibilidad de los valores en un modo cartográfico, homogéneo para diversas localizaciones y aspectos dificulta su uso en una planificación integrada y completa de un territorio. A este respecto, el presente artículo evalúa las posibilidades existentes para trasladar los valores obtenidos por los distintos métodos a un formato cartográfico a un nivel de detalle operativo. No se discute la posibilidad de la obtención de los valores en sí, sino su utilidad práctica para la planificación territorial.

Desde este marco práctico, en el estado actual de aplicación y desarrollo de los métodos, no es posible elaborar una cartografía general de todos los aspectos ambientales que permitiera disponer del Valor Económico Total con unos costes de tiempo y dinero razonables. Los esfuerzos realizados para obtener este VET se han realizado hasta ahora sólo a nivel contable (Campos y Riera, 1996; Croitoru, 2004; Merlo y Croitoru, 2005). Sin embargo, sí pueden cartografiarse ciertos aspectos ambientales.

De hecho, los valores de los bienes ambientales de uso directo puede ser todos cartografiados aún cuando sean bienes de no-mercado (sin precio establecido). Los servicios de uso directo (recreo, caza, paisaje, etc.) pueden ser cartografiados pero aún requieren un mayor desarrollo de los métodos a ese respecto.

Los valores de bienes y servicios de uso indirecto pueden ser cartografiados si lo son las funciones de las que dependen. Aumentan la complejidad de la cartografía y requieren 
tiempos de actualización más rápidos (son más sensibles a los cambios).

Actualmente no es posible realizar una cartografía adecuada a la planificación territorial (regional) que incluya valores de no-uso. Podría plantearse excepcionalmente para la elaboración de cartografías generales a una escala global o mundial (Schuyt y Brander, 2004), pero no para cartografías aplicables a la planificación territorial. Aún cuando se asumiesen los elevados costes y se emprendiese un exhaustivo levantamiento de información, las dificultades metodológicas mencionadas en apartados anteriores sesgarian sin remedio el resultado.

\section{CONCLUSIONES}

Con el actual desarrollo de los métodos no es posible a corto plazo obtener una cartografía económica de todos los aspectos ambientales para su uso en planificación regional. Si bien sí es posible cartografiar la valoración económica ciertos elementos ambientales (algunos valores de uso y opción) para mejorar su integración con el resto de elementos considerados en la planificación territorial.

Pese a esta afirmación, el desarrollo de la línea de investigación actual hacia un mejor conocimiento de los métodos y de los casos es alentador. Si se desarrolla completamente el método de transferencia de resultados (lo cual requiere un número elevado de estudios de diversos métodos y bajo determinadas condiciones que permitan realizar meta-análisis) la obtención de una cartografía económica de todos los aspectos ambientales es perfectamente posible. Es más, la información de que se dispondría con respecto a la relación entre los elementos del territorio y sus valores económicos serían muy útiles para su uso en planificación regional.

\section{REFERENCIAS}

AzQueta, D. (2002). Introducción a la economía ambiental, McGraw Hill / Interamericana de España, Madrid.

Azoueta, D. y Delacamara, G. (2006). "Ethics, economics and environmental management." Ecological Economics, 56(4), 524-533.

Azqueta Oyarzun, D. (2002). Introducción a la Economía Ambiental, McGraw Hill / Interamericana de España.

Batabyal, A. A., Kahn, J. R. y O'NeILl, R. V. (2003). "On the scarcity value of ecosystem services." Journal of Environmental Economics and Management, 46(2), 334.

Bateman, I. J., Cole, M., Cooper, P., Georgiou, S., Hadley, D. y Poe, G. L. "Visible Choice Sets and Scope Sensitivity in Contingent Values.n American Agricultrual Economics Association's Annual Meeting, Chicago, Illinois, 31

Beneitez LóPEZ, J. M., Gómez JiménEZ, I. y Espinal Gómez, F. M. «Metodología de la modelización integral de la planificación recreativa en sistemas forestales." XII Congreso Nacional de Tecnologías de la Información Geográfica. El acceso a la información espacial y las nuevas tecnologías geográficas, Granada, Spain.

CAmpos, P. y Riera, P. (1996). "Rentabilidad social de los bosques: Análisis aplicado a las dehesas y los montados ibéricos." Información Comercial Española, 751, 47-62.
Carson, R. T., Flores, N. E. y Meade, N. F. (2001). "Contingent Valuation: Controversies and Evidence." Environmental and Resource Economics, 19(2), 173-210.

ClARK, J., BuRgESS, J. y HARRISON, C. M. (2000). "I"I struggled with this money business": respondents' perspectives on contingent valuation." Ecological Economics, 33(1), 45.

CRoltoRU, L. "Valuing forest public goods and externalities: an application to Mediterranean forests." /I Simposio Iberoamericano de Gestión y Economía Forestal, Barcelona (Spain).

Chattopadhyay, S. (2003). "A Repeated Sampling Technique in Assessing the Validity of Benefit Transfer in Valuing Non-Market Goods." Land Economics, 79(4), 576-596.

Desvousges, W. H., JOHnson, F. R. y BAnzhaF, H. S. (1998). Environmental Policy Analysis with Limited Information: Principles and Applications of the Transfer Method, Edward Elgar, Cheltenham, UK.

DreCHSLER, M. (2004). "Model-based Conservation Decision Aiding in the Presence of Goal Conflicts and Uncertainty." Biodiversity and Conservation, 13(1), 141-164.

EADE, J. D. O. y MORAN, D. (1996). "Spatial Economic Valuation: Benefits Transfer using Geographical Information Systems." Journal of Environmental Management, 48(2), 97-110.

EKINS, P. (2003). «Identifying critical natural capital: Conclusions about critical natural capital.» Ecological Economics, 44(2-3), 277-292.

EloRRIETA, I. y CASTELLANO, E. (2000). «Valoración integral de la conservación de la biodiversidad de Navarra." Actas del III Congreso de Economía de Navarra.

Farber, S. C., Costanza, R. y Wilson, M. A. (2002). «Economic and ecological concepts for valuing ecosystem services.n Ecological Economics, 41(3), 375-392.

Gómez Jiménez, I., Picher Fernández, A. C. y Romero-Calcerrada, R. «Modelización de la dinámica espacio-temporal de la erosión como instrumento para la toma de decisiones territoriales." XII Congreso Nacional de Tecnologías de la Información Geográfica. El acceso a la información espacial y las nuevas tecnologías geográficas, Granada, Spain.

JAYASURIYA, R. T. (2003). "Measurement of the scarcity of soil in agriculture.n Resources Policy, 29(3-4), 119-129.

Lovet, A. A., Brainard, J. S. y Bateman, I. J. (1997). "Improving Benefit Transfer Demand Functions: A GIS Approach." Journal of Environmental Management, 51(4), 373-389.

MARTinez de Anguita, P. (2004). «Economía ambiental y ordenación del territorio." Ecosistemas, 1.

Merlo, M. y Croltoru, L. (2005). «Valuing Mediterranean Forests. Towards Total Economic Value." Cabi Publishing, Padova, 406.

MilLWARD, H. (1993). "Public access in the West European countryside: a comparative survey." Journal of Rural Studies, 9(1), 39.

MOgAS, J. y RIERA, P. "El valor de la fijación de carbono en los programas de forestación." /I Simposio Iberoamericano de Gestión y Economía Forestal, Barcelona (Spain).

Oglethorpe, D., Hanley, N., Hussain, S. y SANDerson, R. (2000). «Modelling the transfer of the socio-economic benefits of environmental management." Environmental Modelling and Software with Environment Data News, 15(4), 343.

PAgIOLA, S., Von RITTER, K. y BISHOP, J. (2004). "Assessing the Economic Value of Ecosystem Conservation." E. DEPARTMENT, ed., WORLD BANK, 64.

Parsons, G. R. y Keraly, M. J. (1994). "Benefits transfer in a random utility model of recreation." Water Resources Research, 30(8), 24772484.

PeARCE, D. y Moran, D. (1994). The economic value of biodiversity, Earthscan, London

PeARCE, D. W. y TuRner, R. K. (1991). Economia delle risorse naturali e deIl'ambiente, M. Botticini, translator, il Mulino.

Prada Blanco, A., Vázquez Rodríguez, M. X. y Soliño Millán, M. (2005). Beneficios y costes sociales en la conservación de la Red Natura 2000, CIEF (Centro de Investigación Económica y Financiera), Fundación Caixa Galicia. 
PukKala, T., NuUtinen, T. y Kangas, J. (1995). «Integrating scenic and recreational amenities into numerical forest planning." Landscape and Urban Planning, 32(3), 185-195.

RierA, P. (1992). «Posibilidades y Limitaciones del Instrumental Utilizado en la Valoración de Externalidades.» ICE.

RIERA, P. y MOgAS, J. (2004). "Evaluation of a risk reduction in forest fires in a Mediterranean region." Forest Policy and Economics, 6(6), 521-528.

SChuYt, K. y BRAnder, L. (2004). "The Economic Values of the World's Wetlands." WWF, Gland/Amsertadam.

SPASH, C. L. (2000). "Ecosystems, contingent valuation and ethics: the case of wetland re-creation." Ecological Economics, 34(2), 195215 .
TYRVAinen, L. (2001). «Economic valuation of urban forest benefits in Finland." Journal of Environmental Management, 62(1), 75-92.

TYRVAINEN, L. y VAANANEN, H. (1998). "The economic value of urban forest amenities: an application of the contingent valuation method." Landscape and Urban Planning, 43(1), 105.

VAn NoORdWiJk, M., Poulsen, J. G. y ERICKSEN, P. J. (2004). "Quantifying off-site effects of land use change: filters, flows and fallacies.n Agriculture, Ecosystems \& Environment(104), 19-34.

VeNKATACHALAM, L. (2004). "The contingent valuation method: a review." Environmental Impact Assessment Review, 89-124.

Villa, F., Wilson, M. A., de Groot, R., Farber, S., Costanza, R. y Boumans, R. M. J. (2002). "Designing an integrated knowledge base to support ecosystem services valuation." Ecological Economics, 41(3), 445-456. 